\title{
Distinct 8-QAM+ Perfect Arrays
}

\author{
Fanxin Zeng ${ }^{1, a}$, Zhenyu Zhang ${ }^{2,1, ~ b}$, Linjie Qian ${ }^{1, c}$ \\ ${ }^{1}$ Chongqing Key Laboratory of Emergency Communication, Chongqing Communication Institute, \\ Chongqing, 400035, China \\ ${ }^{2}$ College of Communication Engineering, Chongqing University, Chongqing, 400044, China \\ email: afzengx@163.com, 'bhenyu.zhang@cqu.edu.cn, ${ }^{\mathrm{c}}$ charlige@126.com
}

Keywords: perfect arrays; 8-QAM+ constellation; family size

\begin{abstract}
This paper investigates the construction of 8-QAM+ perfect arrays with at least an odd integer in their sizes, and gives the sufficient condition producing distinct 8-QAM+ perfect arrays. For different choices of odd integers in a given size of at least an odd integer, the resultant distinct 8-QAM+perfect arrays have their own sizes. For each choice, the number of the resultant distinct 8-QAM+perfect arrays is exactly equivalent to this chosen odd integer. This paper can provide more candidates for the applications of 8-QAM+ perfect arrays in communications, radar, and so forth.
\end{abstract}

\section{Introduction}

Perfect arrays have the property of impulse-like autocorrelation, which results in the fact that perfect arrays are widely applied to communications, radar, time-frequency-coding, map matching, synchronization, built-in tests of VLSI-circuits, and so on [1]. There are a large number of perfect binary, ternary, and ployphase array [1]. However, 8-QAM+ perfect arrays are rare, whose main reason lies at the late introduction of 8-QAM+ constellation [2]. In 2010, Boztas and Parampalli introduced the definition of 8-QAM+ signal set [2]. From that time on, the sequences over 8-QAM+ constellation are rapidly developed. Perfect and odd perfect 8-QAM+ sequences are given in 2012 and 2014 , respectively [3] [4]. 8-QAM+ complementary sequences are presented in 2012 and 2014, respectively [5] [6]. ZCZ 8-QAM+ sequences appeared in 2012 [7]. Recently, 8-QAM+ perfect arrays are proposed in 2014 and 2015 [8] [9].

The aim of this paper is to investigate 8-QAM+ perfect arrays, to increase their family size, and to reflect the state-of-the-art of this area. A sufficient condition producing distinct 8-QAM+ perfect arrays will be introduced. The number of the resultant 8-QAM+ perfect arrays will be improved.

The rest of this paper will organized as follows. In Section 2, we will review some necessary concepts and the known results on the constructions of 8-QAM+ perfect arrays. In Section 3, a sufficient condition producing distinct 8-QAM+ perfect arrays will be given. The examples will appear in Section 4. Finally, we conclude this paper in Section 5.

\section{Necessary Concepts and Known Constructions of 8-QAM+ Perfect Arrays}

In a mathematical term, an array $A$ of $n$-dimension with the size $N_{1} \times N_{2} \times \cdots \times N_{n}$ is

$$
A=\left[a_{i_{1}, i_{2}, \cdots, i_{n}} \mid 0 \leq i_{k}<N_{k}, 1 \leq k \leq n\right],
$$

whose periodic autocorrelation is defined by

$$
R_{A, A}\left(\tau_{1}, \tau_{2}, \cdots, \tau_{n}\right)=\sum_{i_{1}=0}^{N_{1}-1} \sum_{i_{2}=0}^{N_{2}-1} \cdots \sum_{i_{n}=0}^{N_{n}-1} a_{i_{1}, i_{2}, \cdots, i_{n}}\left[a_{i_{1}+\tau_{1}, i_{2}+\tau_{2}, \cdots, i_{n}+\tau_{n}}\right]^{*},
$$

where the symbol $x^{*}$ denotes the complex-conjugate of $x$, and the subscript addition $i_{k}+\tau_{k}$ $(1 \leq k \leq n)$ is performed modulo $N_{k}$.

If the autocorrelation of an array $A$ of $n$-dimension satisfies 


$$
R_{A, A}\left(\tau_{1}, \tau_{2}, \cdots, \tau_{n}\right)=\left\{\begin{array}{ll}
>0 & \left(\tau_{1}, \tau_{2}, \cdots, \tau_{n}\right)=(0,0, \cdots, 0) \\
0 & \left(\tau_{1}, \tau_{2}, \cdots, \tau_{n}\right) \neq(0,0, \cdots, 0)
\end{array},\right.
$$

we refer to this array as perfect array.

Let the symbol $T$ denote the $n$-dimensional cyclical shift operator, in other words, for a given array, say $A$ in (1), and given $n$ integers $\mu_{k}$ 's $(1 \leq k \leq n)$, under this operator a new array $T\left(\mu_{1}, \mu_{2}, \cdots, \mu_{n}\right) A$ can be given by

$$
T\left(\mu_{1}, \mu_{2}, \cdots, \mu_{n}\right) A=\left[a_{i_{1}+\mu_{1}, i_{2}+\mu_{2}, \cdots, i_{n}+\mu_{n}} \mid 0 \leq i_{k}<N_{k}, 1 \leq k \leq n\right],
$$

where the subscript addition $i_{k}+\mu_{k} \quad(1 \leq k \leq n)$ is counted modulo $N_{k}$.

Let $A$ and $B$ be two arrays with the same size. If there exist $n$ integers $\mu_{k}$ 's $(1 \leq k \leq n)$ so that

$$
T\left(\mu_{1}, \mu_{2}, \cdots, \mu_{n}\right) A=B,
$$

we say these two arrays to be equivalent, or else, to be distinct.

The 8-QAM+ constellation means the signal set [2]:

$\Omega_{8}^{+}=\{0,-1,1,-j, j,-1-j, 1+j, 1-j,-1+j\}$.

Based on the direct product $\{-1,0,1\} \times\{-1,0,1\}$, the 8 -QAM+ constellation can be produced by the following mappings: [3]

$$
\begin{aligned}
\pi:\{-1,0,1\} \times\{-1,0,1\} & \rightarrow \Omega_{8}^{+} \\
(a, b) & \rightarrow \pi(a, b),
\end{aligned}
$$

where

$$
\pi_{1}(a, b)=a j+b \quad \text { and } \quad \pi_{2}(a, b)=a j-b .
$$

By the aforementioned mappings, a ternary array can be converted an 8-QAM+array [8] [9]. The construction methods referred to in this paper are given by the following two Theorems.

Theorem 1 [8] [9]: Let $A$ be the perfect ternary array (PTA) with the size $N_{1} \times N_{2} \times \cdots \times N_{n}$, and $\eta_{k}$ and $\delta_{k} \quad(1 \leq k \leq n)$ are the integers satisfying $-N_{k}+1 \leq \eta_{k}, \delta_{k} \leq N_{k}-1$. If all the integers $N_{k}$ 's $(1 \leq k \leq n)$ are even, and we have

$$
\eta_{k} \equiv \delta_{k}\left(\bmod N_{k} / 2\right) \quad(1 \leq k \leq n),
$$

the 8-QAM+ array $Q=\left[q_{i_{1}, i_{2}, \cdots, i_{n}} \mid 0 \leq i_{k}<N_{k}, 1 \leq k \leq n\right]$ with the size $N_{1} \times N_{2} \times \cdots \times N_{n}$ is perfect, where

$$
q_{i_{1}, i_{2}, \cdots, i_{n}}=\pi_{1}\left(a_{i_{1}+\eta_{1}, i_{2}+\eta_{2}, \cdots, i_{n}+\eta_{n}}, a_{i_{1}+\delta_{1}, i_{2}+\delta_{2}, \cdots, i_{n}+\delta_{n}}\right) .
$$

Theorem 2 [8] [9]: Let $A$ and $B$ be two PTAs with the same size $N_{1} \times N_{2} \times \cdots \times N_{n}$, where at least an integer in the set $\left\{N_{k} \mid 1 \leq k \leq n\right\}$ is odd, say, $N_{r}$. Set the integers $\eta_{k}$ 's, $\delta_{k}$ 's, $\zeta_{k}$ 's, and $\lambda_{k}$ 's $(1 \leq k \leq n)$ satisfy $-N_{k}+1 \leq \eta_{k}, \delta_{k}, \zeta_{k}, \lambda_{k} \leq N_{k}-1$. Construct the 8-QAM+ array $P$ below.

$$
\left\{\begin{array}{l}
P=\left[p_{i_{1}, i_{2}, \cdots, i_{r-1}, i_{r}^{\prime}, i_{r+1}, \cdots, i_{n}} \mid 0 \leq i_{k}<N_{k}, 1 \leq k \leq n, i_{r}^{\prime}=2 i_{r}+t, t \in\{0,1\}\right] \\
p_{i_{1}, i_{2}, \cdots, i_{r-1}, i_{r}^{\prime}, i_{r+1}, \cdots, i_{n}}=\pi_{1}\left(a_{i_{1}+\eta_{1}, \cdots, i_{r-1}+\eta_{r-1}, i_{r}+\eta_{r}, i_{r+1}+\eta_{r+1}, \cdots, i_{n}+\eta_{n}}, b_{i_{1}+\delta_{1}, \cdots, i_{r-1}+\delta_{r-1}, i_{r}+\delta_{r}, i_{r+1}+\delta_{r+1}, \cdots, i_{n}+\delta_{n}}\right) \text { if } i_{r}^{\prime}=2 i_{r} \\
p_{i_{1}, i_{2}, \cdots, i_{r-1}, i_{r}^{\prime}, i_{r+1}, \cdots, i_{n}}=\pi_{2}\left(a_{i_{1}+\zeta_{1}, \cdots, i_{r-1}+\zeta_{r-1}, i_{r}+\zeta_{r}, i_{r+1}+\zeta_{r+1}, \cdots, i_{n}+\zeta_{n}}, b_{i_{1}+\lambda_{1}, \cdots, i_{r-1}+\lambda_{r-1}, i_{r}+\lambda_{r}, i_{r+1}+\lambda_{r+1}, \cdots, i_{n}+\lambda_{n}}\right) \text { if } i_{r}^{\prime}=2 i_{r}+1
\end{array}\right.
$$

If we have

$$
\begin{cases}\delta_{k}-\eta_{k} \equiv \lambda_{k}-\zeta_{k} & \bmod N_{k}(1 \leq k \leq n) \\ \zeta_{k}-\delta_{k} \equiv \eta_{k}-\lambda_{k} & \bmod N_{k}(1 \leq k \leq n, k \neq r), \\ \zeta_{r}-\delta_{r} \equiv \eta_{r}-\lambda_{r}+1 & \bmod N_{r}\end{cases}
$$

the 8-QAM+ array $P$ with the size $N_{1} \times \cdots \times N_{r-1} \times 2 N_{r} \times N_{r+1} \times \cdots \times N_{n}$ is perfect.

Theorem 3 [8] [9]: The equation system (11) has the solution: 


$$
\left\{\begin{array}{ll}
\zeta_{k} \equiv \eta_{k}\left(\bmod N_{k}\right) & \forall \eta_{k}(1 \leq k \leq n, k \neq r) \\
\lambda_{k} \equiv \delta_{k}\left(\bmod N_{k}\right) & \forall \delta_{k}(1 \leq k \leq n, k \neq r) \\
\zeta_{r} \equiv \eta_{r}+\frac{N_{r}+1}{2}\left(\bmod N_{r}\right) & \forall \eta_{r} \\
\lambda_{r} \equiv \delta_{r}+\frac{N_{r}+1}{2}\left(\bmod N_{r}\right) & \forall \delta_{r}
\end{array} .\right.
$$

\section{Production Procedure of New Arrays}

In this section, we investigate the construction method producing distinct 8-QAM+ perfect arrays, based on Theorem 2. Assume we need such arrays with the size $N_{1} \times \cdots \times N_{r-1} \times 2 N_{r} \times N_{r+1} \times \cdots \times N_{n}$, where $N_{r}$ is odd number. The distinct 8-QAM+ perfect arrays can be educed by following the given steps below.

Step 1: Choose two PTAs $A$ and $B$ with the size $N_{1} \times \cdots \times N_{r-1} \times N_{r} \times N_{r+1} \times \cdots \times N_{n}$;

Step 2: Initialization. Arbitrarily choose the integers $\eta_{k}$ 's, $\delta_{k}$ 's, $\zeta_{k}$ 's, and $\lambda_{k}$ 's $(1 \leq k \leq n, k \neq r)$;

Step 3: Initialization. Arbitrarily choose the integers $\delta_{k}$ 's $(1 \leq k \leq n)$;

Step 4: Initialization. Determine the integer $\lambda_{r}$ by $\lambda_{r} \equiv \delta_{r}+\frac{N_{r}+1}{2}\left(\bmod N_{r}\right)$;

Step 5: Iteration times: $t \leftarrow 1$;

Step 6: Set $\eta_{r}=t-1$;

Step 7: Determine the integer $\zeta_{r}$ by $\zeta_{r} \equiv \eta_{r}+\frac{N_{r}+1}{2}\left(\bmod N_{r}\right)$;

Step 8: Produce 8-QAM+ array $P$, denoted by $P_{t}$, by Eq. (11) in Theorem 2;

Step 9: If $t \leq N_{r}-1$, turn to Step 6 with $t \leftarrow t+1$, or else turn to Step 10;

Step 10: End.

Summarizing the above, we can obtain $N_{r}$ distinct 8-QAM+ perfect arrays. Incidentally, for the reader who wants to know the detailed derivation of this procedure, please refer to [8].

\section{Examples}

Consider the production of 8-QAM+ perfect arrays with the size $2 \cdot 3 \times 7$. Choose the PTA with the size $3 \times 7$ below.

$$
\left[\begin{array}{ccccccc}
1 & 0 & 0 & 1 & 0 & 1 & 1 \\
0 & 1 & 1 & -1 & 1 & -1 & -1 \\
0 & 1 & 1 & -1 & 1 & -1 & -1
\end{array}\right]
$$

Note that $r=1$. In accordance with our steps, we initialize that $\left(\delta_{1}, \delta_{2}\right)=(1,0),\left(\eta_{2}, \zeta_{2}\right)=(0,0)$, and $\left(\lambda_{1}, \lambda_{2}\right)=(0,0)$. Now, we perform our procedure as follows.

(1) When the iteration times $t=1$, we have $\left(\eta_{1}, \zeta_{2}\right)=(0,2)$. The resultant 8 -QAM+ perfect array is

$$
P_{0}=\left[\begin{array}{ccccccc}
j & 1 & 1 & -1+j & 1 & -1+j & -1+j \\
-1 & j & j & -1-j & j & -1-j & -1-j \\
0 & 1+j & 1+j & -1-j & 1+j & -1-j & -1-j \\
j & -1 & -1 & 1+j & -1 & 1+j & 1+j \\
1 & j & j & 1-j & j & 1-j & 1-j \\
0 & -1+j & -1+j & 1-j & -1+j & 1-j & 1-j
\end{array}\right] .
$$

(2) When the iteration times $t=2$, we have $\left(\eta_{1}, \zeta_{2}\right)=(1,0)$. The resultant 8-QAM+ perfect array is given by 


$$
P_{1}=\left[\begin{array}{ccccccc}
0 & 1+j & 1+j & -1-j & 1+j & -1-j & -1-j \\
-1+j & 0 & 0 & -1+j & 0 & -1+j & -1+j \\
0 & 1+j & 1+j & -1-j & 1+j & -1-j & -1-j \\
0 & -1+j & -1+j & 1-j & -1+j & 1-j & 1-j \\
1+j & 0 & 0 & 1+j & 0 & 1+j & 1+j \\
0 & -1+j & -1+j & 1-j & -1+j & 1-j & 1-j
\end{array}\right] .
$$

(3) When the iteration times $t=3$, we have $\left(\eta_{1}, \zeta_{2}\right)=(2,1)$. The resultant 8-QAM+ perfect array is equivalent to

$$
P_{2}=\left[\begin{array}{ccccccc}
0 & 1+j & 1+j & -1-j & 1+j & -1-j & -1-j \\
-1 & j & j & -1-j & j & -1-j & -1-j \\
j & 1 & 1 & -1+j & 1 & -1+j & -1+j \\
0 & -1+j & -1+j & 1-j & -1+j & 1-j & 1-j \\
1 & j & j & 1-j & j & 1-j & 1-j \\
j & -1 & -1 & 1+j & -1 & 1+j & 1+j
\end{array}\right] .
$$

The autocorrelation functions of all the arrays $P_{0}-P_{2}$ are depicted in Fig.1 below. Apparently, it is an impulse-like function as promised in our procedure.

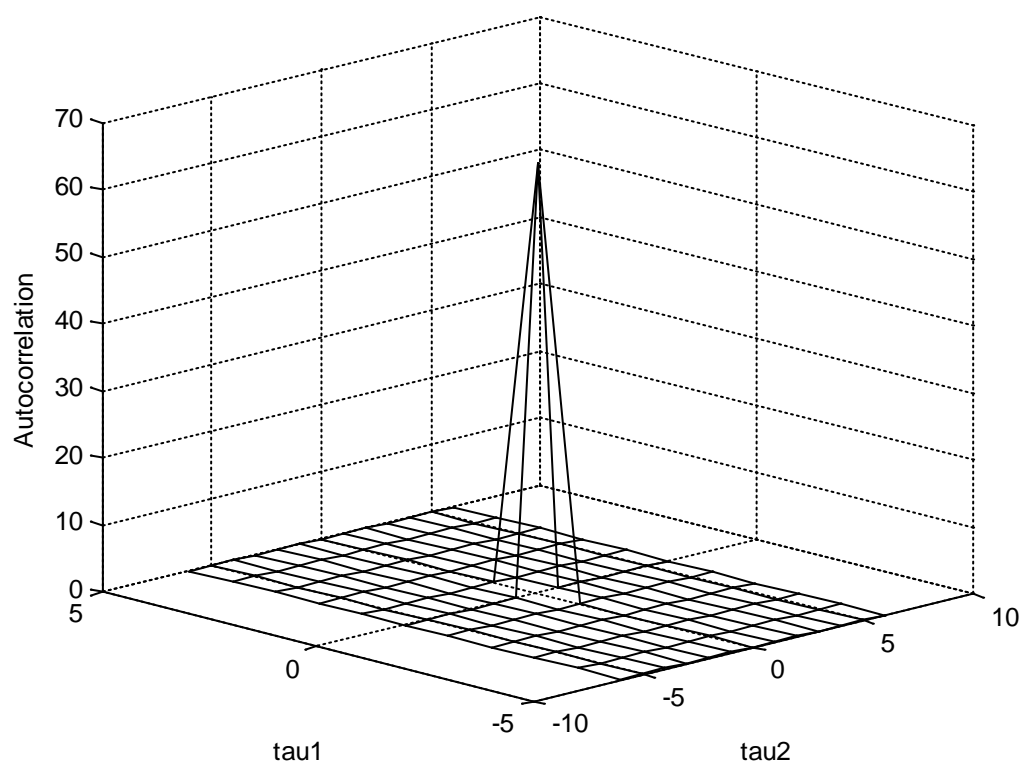

Fig.1 Autocorrelation of the resultant 8-QAM+ perfect arrays.

\section{Conclusion}

This paper gives the production procedure of 8-QAM+ perfect arrays, and some examples show its validation. However, the flaw of this procedure lies at the expansion of the size of the resultant arrays, in comparison with the size of the arrays employed. It is our future work to explore new method so as to construct the 8-QAM+ perfect arrays with the size unaltered.

\section{Acknowledgement}

This work was supported by Chongqing Science and Technology Committee (cstc2014pt-sy40003), and National Natural Science Foundation of China (NSFC) under Grants 60872164, 61002034, 61271003, and 61471336, China Postdoctoral Science Foundation Grant 2014M552318, Natural Science Foundation Project of CQ Grant cstc2014jcyj A40050, and 
Chongqing Postdoctoral Science Special Foundation Grant Xm2014031.

\section{References}

[1] Pingzhi Fan, Michael Darnell. Sequence design for communications applications. John Wiley \& Sons INC., 1996, Ch. 12.

[2] Serdar Boztas, Udaya Parampalli. Nonbinary sequences with perfect and nearly perfect autocorrelations. Proceedings International Symposium on Information Theory, Austin, Texas, U.S.A., 2010, 1300-1304.

[3] Fanxin Zeng, et al. Perfect 8-QAM+ sequences. IEEE Wireless Commun. Lett., 2012 1(4) 388-391.

[4] Yubo Li, et al. Odd perfect sequences and sequence sets with zero odd correlation zone over the 8-QAM+ constellation. IEICE Trans. Fundamentals, 2014 E97-A(1) 425-428.

[5] Fanxin Zeng, et al. 8-QAM+ periodic complementary sequence sets, IEEE Commun. Lett., 2012 16(1) 83-85.

[6] Fanxin Zeng, et al. A note on 8-QAM+ sequences, IEICE Trans. Fundamentals, 2104 E97-A(3) 888-893.

[7] Yubo Li, et al. Zero correlation zone sequence sets over the 8-QAM+ constellation. IEEE Commun. Lett., 2012 16(11) 1844-1847.

[8] Fanxin Zeng, et al. Perfect arrays over the 8-QAM+ constellation. IEICE Trans. Fundamentals. 2015 E98-A(4) 1038-1043.

[9] Fanxin Zeng, et al. Design of 8-QAM+ perfect arrays from perfect ternary arrays. Proceedings 2014 7th International Congress on Image and Signal Processing, Dalian, China, 2014, 1185-1190. 